\title{
Simulation of the electron-cloud build up and its consequences on heat load, beam stability, and diagnostics
}

\author{
G. Rumolo,* F. Ruggiero, and F. Zimmermann \\ CERN, SL Division, CH-1211 Geneva 23, Switzerland \\ (Received 9 October 2000; published 8 January 2001)
}

\begin{abstract}
Photoemission and secondary emission are known to give rise to a quasistationary electron cloud inside the beam pipe through a beam-induced multipacting process. We investigate the electron-cloud build up and related effects via computer simulation. In our model, macroparticles representing photoelectrons are emitted synchronously with the passing proton or positron bunch and are subsequently accelerated in the field of the beam. As they hit the beam pipe, new macroelectrons are generated, whose charges are determined by the energy of the incoming particles and by the secondary emission yield of the beam pipe. A quasistationary state of the electron cloud is eventually reached due to space charge. The equilibrium density is used as an input parameter for a second program that analyzes the electron-cloud driven single-bunch instability. The electron cloud simulation also allows the evaluation of the heat load on the cold Large Hadron Collider beam screen, which must stay within the available cooling capacity, and the electron charge deposited on or emitted from the electrodes of the beam-position monitors.
\end{abstract}

DOI: $10.1103 /$ PhysRevSTAB.4.012801

PACS numbers: 41.75.Ht, 29.27.Bd

\section{INTRODUCTION}

Photoemission and electron multiplication on surfaces exposed to an oscillating electromagnetic field cause the phenomenon of multipacting, which can significantly degrade the performance of rf cavities as well as that of storage rings operating with closely spaced positron or proton bunches.

Beam-induced multipacting was observed as a pressure rise at the CERN intersection storage ring (ISR) in 1977, after installation of an aluminum test chamber [1]. Based on the ISR experience, concerns about the Large Hadron Collider (LHC) operation started in the 1980's [2]. In 1989, an instability at the KEK Photon Factory was attributed to photoelectrons [3,4]. In 1996, a series of electron-cloud experiments were conducted by an IHEP-KEK Collaboration at the Beijing Electron Positron Collider [5]. Shortly thereafter, crash programs were launched for the positron ring (LER) of the PEP-II B Factory [6,7] (simulations, TiN coating of $\mathrm{Al}$ vacuum chamber) and, after simulations and analytical estimates had predicted a serious effect for heat load and beam stability [8,9], for LHC [10-15]. The possibility of beam-induced multipacting in the LHC was first mentioned by Gröbner in 1996 [2]. Since 1998, electron-cloud effects have been seen with the LHC test beam in the superprotron ring (SPS) as well (see the session on electron cloud in Ref. [16]).

As schematically illustrated in Fig. 1, the electron-cloud build up in the vacuum chamber results from a combination of processes. Each passing bunch generates a number

*Corresponding author. Telephone: +41-22-7679048. Fax: +41-22-7830552. Email address: Giovanni.Rumolo@cern.ch of primary electrons (generally referred to as photoelectrons), which are later accelerated by the beam field and may create secondary electrons at their impact with the vacuum chamber. If the energy of the incoming electrons is sufficiently large, the secondary emission yield (SEY) is greater than unity, and the number of electrons grows exponentially. The electron-cloud build up stops at a density roughly equal to the neutralization density [8], where the attractive force from the beam is on average balanced by the space charge repulsion of the electron cloud.

Primary electrons are needed to initiate the build up of the electron cloud. In the LHC a large number of primary electrons are generated via photoemission from synchrotron radiation. The rate of incident photons is [17]

$$
N_{\gamma}=\frac{5}{2 \sqrt{3}} \alpha \gamma \frac{\text { photons }}{\text { radians }} \quad \text { or } \quad 0.025 \frac{\text { photons }}{\text { proton } \mathrm{m}}
$$

where $\gamma$ is the relativistic factor of the irradiating beam and $\alpha$ is the fine-structure constant. The critical photon energy is $E_{c} \simeq 45 \mathrm{eV}$. The measured data show that at this energy the photoemission yield is close to maximum $[18,19]$.

The photon reflectivity is another important parameter. If the reflectivity of the vacuum chamber is high, many photoelectrons are created at the top and bottom of the chamber. On the other hand, if the reflectivity is low, the majority of the photoelectrons impinges on the horizontally outward side of the vacuum chamber. In dipole magnets, these electrons do not approach the beam, and they stay at fairly low energies.

The electron yield per absorbed photon can be expressed as $Y^{*}=Y /(1-R) \simeq 0.05$ where $Y$ is the photoelectron yield per incident photon and $R$ is the photon reflectivity. 


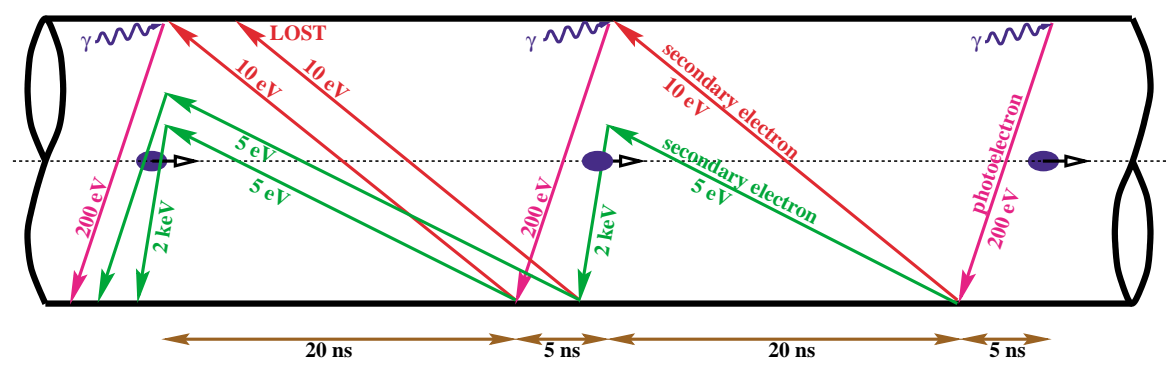

FIG. 1. (Color) Schematic of electron-cloud build up in the LHC beam pipe.

Inserting numbers for $\mathrm{LHC}$ at $7 \mathrm{TeV}$, one can estimate that the creation rate is

$$
\frac{d \lambda_{e}}{d s} \simeq 10^{-3} \frac{\text { photoelectrons }}{\text { proton } \mathrm{m}} .
$$

The beam-induced electron cloud can produce a serious heat load in the LHC beam screen and it may also give rise to a perturbation of the beam position monitor (BPM) signals due to unequal electron bombardment.

In the following, we will first describe how the electroncloud build up is modeled and simulated (Sec. II), discussing, in particular (relative subsections), the associated heat load on the beam screen in the LHC bending magnets and the consequent charge flux into or from the electrodes of a beam position monitor placed along the LHC beam path. Then, we will describe a second macroparticle simulation code modeling the interaction between bunch and electrons, in order to study fast single-bunch instabilities due to the electron cloud (Sec. III). The application of this model to the low energy ring of the KEK B Factory allows us to discuss the effects of this transverse instability in terms of beam centroid oscillation and emittance growth. Finally, Sec. IV summarizes the results and draws an outline for future work and development.

\section{SIMULATION MODEL}

The simulation recipe is illustrated in Fig. 2. The electrons are represented by macroparticles. Typically 5001000 of these are generated per bunch passage. Both bunches and interbunch gaps are split into slices. For each bunch slice, photoelectrons are created and existing electrons are accelerated in the field of the beam, the electrons, and the image charges. Whenever an electron hits the wall, it may generate secondary electrons. In the simulation, the incident macroparticle is reemitted with a different charge. When the secondary emission yield is very high, more secondary electrons are launched, such that the charge of each of them is less or equal to that of the primary electrons: this prevents the undesirable situation where most of the electron charge is carried by very few macroparticles. During the interbunch gap, the electrons are propagated in the magnetic field. Between gap slices, kicks are applied which model the effect of electron space charge and electron image charges.

Simulation results are (i) the energy of the lost electrons, which eventually translates into a heat load, (ii) the number of electrons drifting to and from the electrodes of a pickup monitor, which allows evaluation of the amount of charge deposited on each plate and the relative current, and (iii) the equilibrium electron-cloud density in the neighborhood of the beam, which is the main input for the study of the single-bunch instability.

The photoelectrons are emitted with an initial azimuthal and energy distribution as shown in Figs. 3 and 4. For a reflectivity factor $R$, a fraction $(1-R)$ of the photons are unreflected. In the simulation, they produce photoelectrons within an outward cone of rms angle $11.25^{\circ}$ centered around the horizontal plane. The remainder is emitted around the chamber with a distribution that can be chosen according to the simulation requirements: for the originally designed LHC chamber (with smooth inner walls), a uniform distribution of the reflected light was assumed, whereas the sawtooth version of the chamber requires different types of reflected photon distribution to be used,

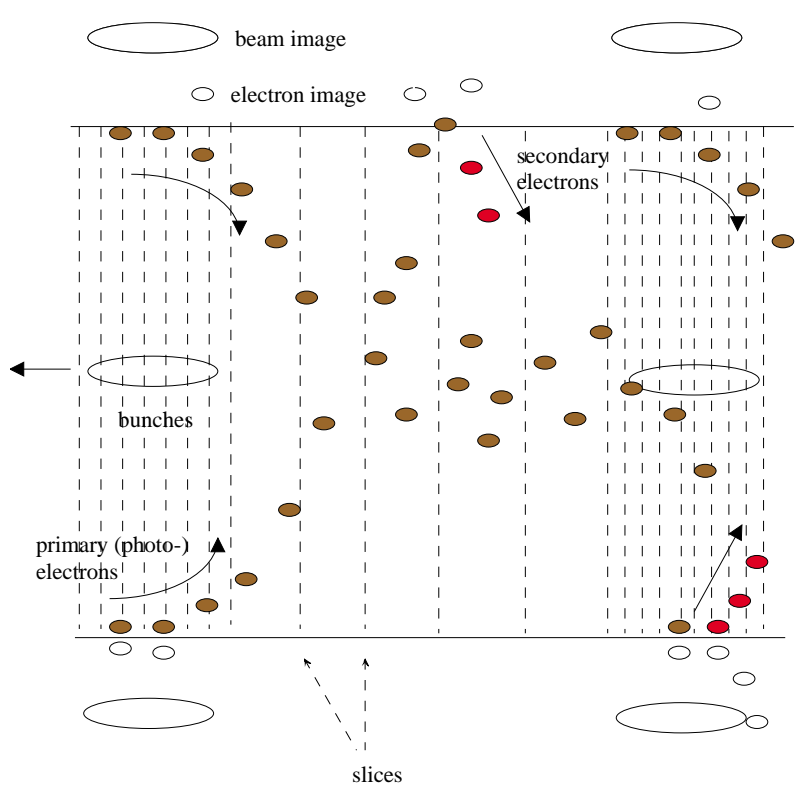

FIG. 2. (Color) Schematic of simulation recipe. 

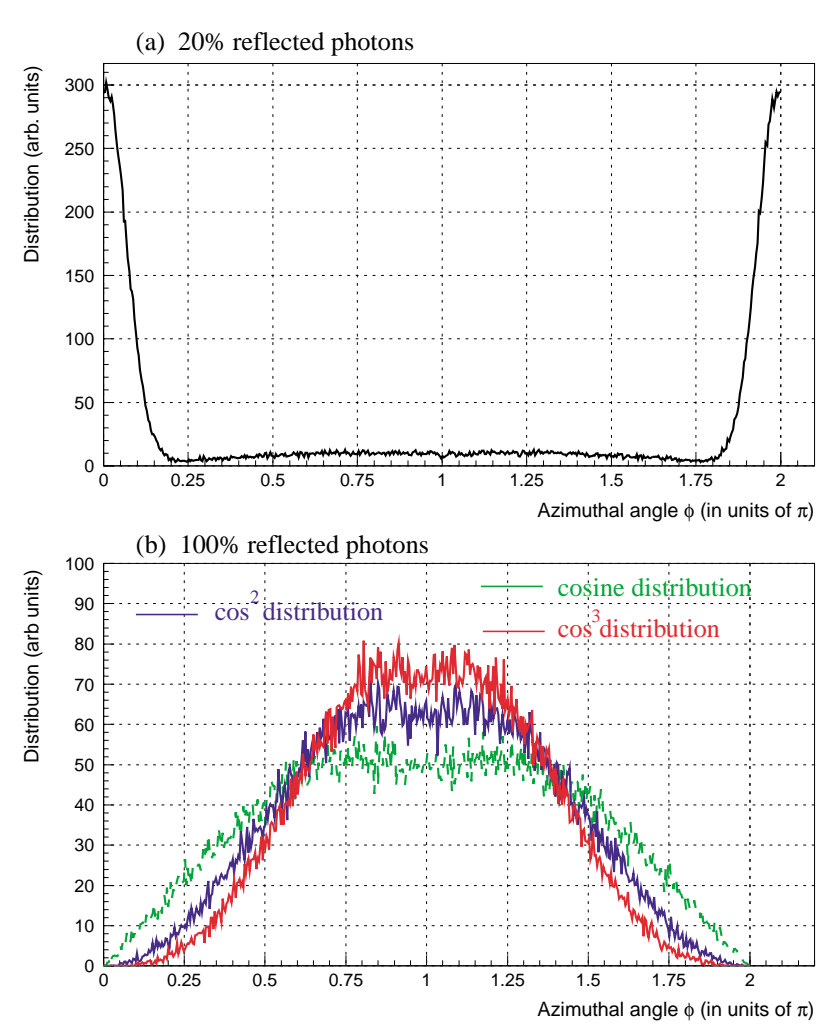

FIG. 3. (Color) Initial azimuthal distribution of photoelectrons for $20 \%$ (a) and $100 \%$ (b) photon reflectivity.

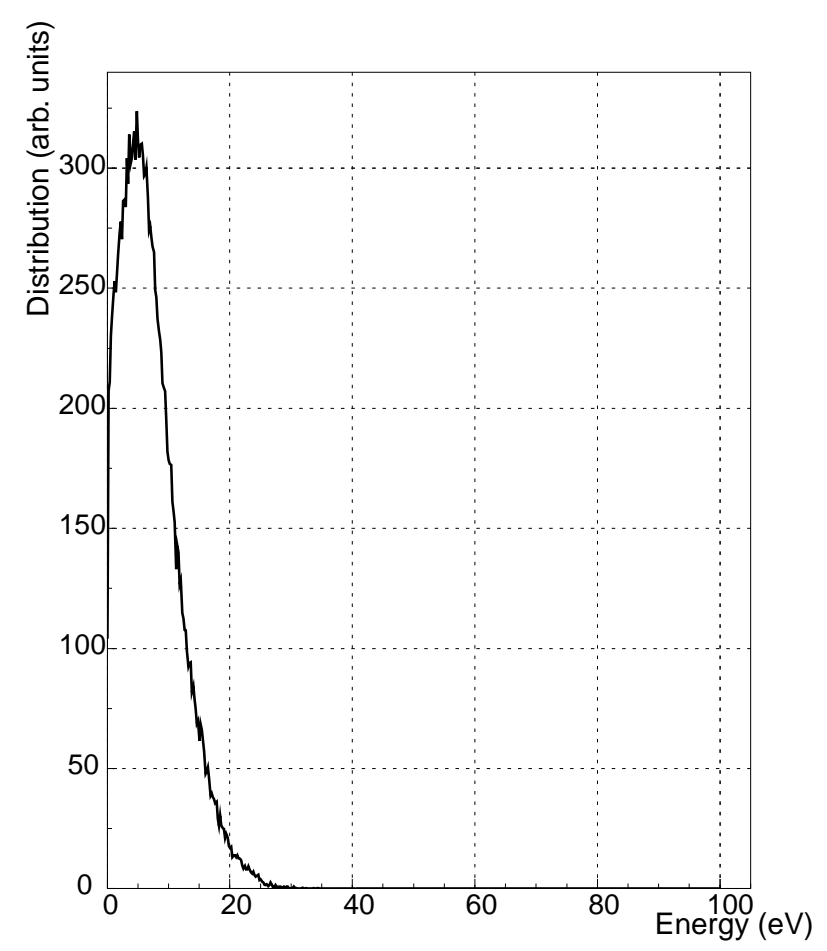

FIG. 4. Initial photoelectron energy distribution at the moment of emission. such as a cosine squared or even cosine cubed distribution [20]. The total azimuthal distribution is shown in Fig. 3 for different shapes and for two different values of $R$. The initial energy distribution of the photoelectrons is chosen as a Gaussian with a peak at $7 \mathrm{eV}$ and rms spread of $5 \mathrm{eV}$ (Fig. 4). Since the photoelectrons are emitted at the time when the generating bunch passes by with a time distribution equal to the bunch distribution, most of them are immediately accelerated in the beam field. The bunch imparts to an electron at the chamber wall a maximum energy of

$$
E_{\max }=2 m_{0} c^{2}\left(N_{b} r_{e} / b\right)^{2} \simeq 200 \mathrm{eV},
$$

where $N_{b}$ is the number of particles per bunch and $b$ is the radius of the vacuum chamber. The electron motion has been simulated for a (1) drift space, (2) strong dipole field, (3) weak dipole, (4) solenoid, (5) quadrupole, (6) arbitrary fields, with a Runge-Kutta integration of the equations of motion [11], and (7) wire and coaxial chambers used in laboratory tests [21].

In a strong (vertical) dipole field, the electron motion is constrained to the vertical direction. In the simulation, we ignore the cyclotron motion and the electron receives only a net vertical kick from the passing bunches. The horizontal kick is approximately 0 due to the large number of cyclotron oscillations performed during the bunch passage. For example, using LHC parameters, the number of cyclotron oscillations is about

$$
\frac{e B c}{m_{e} c^{2}} \frac{2 \sigma_{z}}{2 \pi} \simeq 120 \text {. }
$$

During a bunch passage, an electron may either receive a single kick or perform many oscillations in the bunch potential, depending on its initial position, as illustrated in Fig. 5. For each of the two limiting cases, the maximum energy transfer can be calculated, with results as shown in Fig. 6 [12].

Whenever an electron is lost to the wall, it may generate one or more secondary electrons. The average number of

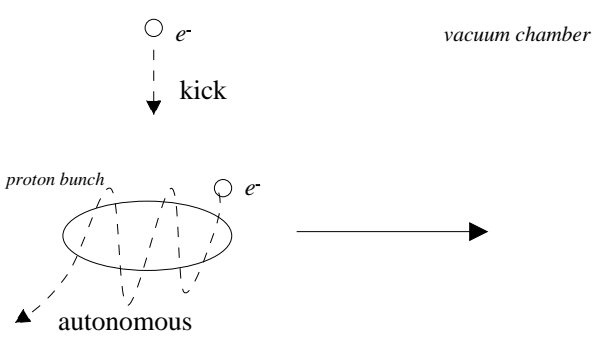

vacuum chamber

FIG. 5. Electrons at large amplitudes do not move much during the bunch passage and they simply receive a kick. Electrons near the bunch oscillate in the beam potential. 


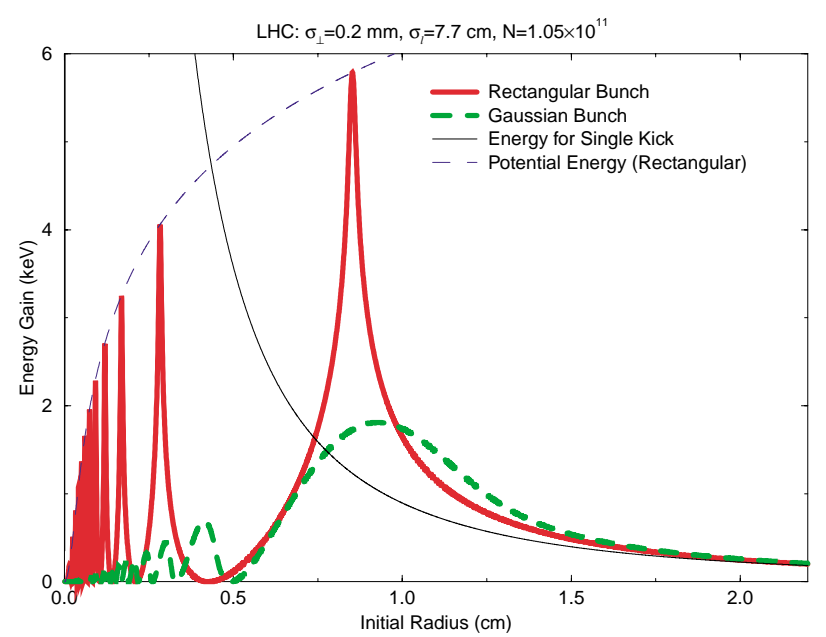

FIG. 6. (Color) Maximum energy gain vs initial particle radius for nominal LHC parameters [12].

secondaries per incident electron has been described by a universal curve [22], which is characterized by only two material parameters: the maximum secondary emission yield for perpendicular incidence, $\delta_{\max }$, and the energy at which this maximum occurs, $\epsilon_{\max }$.

Introducing the angle of incidence with respect to the surface normal, $\theta$, and the normalized electron energy $x=E_{p} / \epsilon_{\mathrm{max}}$, the analytical expression for the secondary emission yield reads [22]

$$
\begin{aligned}
\delta_{\text {se }}\left(E_{p}, \theta\right)= & \delta_{\max } 1.11 x^{-0.35}\left[1-\exp \left(-2.3 x^{1.35}\right)\right] \\
& \times \exp \left(\frac{1-\cos \theta}{2}\right) .
\end{aligned}
$$

An additional yield component represents elastic reflections of the incident electrons. This can be parametrized as [23]

$\delta_{e}\left(E_{p}\right)=\delta_{e, \infty}+\left(\hat{\delta}_{e}-\delta_{e, \infty}\right) \exp \left[-\frac{\left(E_{p}-E_{e}\right)^{2}}{2 \Delta^{2}}\right]$,

with, e.g., $\hat{\delta}_{e}=0.1, \delta_{e, \infty}=0.02$, and $\Delta=E_{e}=5 \mathrm{eV}$ (the numbers come from Ref. [23], but see also the next subsection).

The universal curve, Eq. (5), is illustrated in Figs. 7(a) and 7(b). The additional curve in Fig. 7(a) includes the additional contribution from elastic reflections, Eq. (6).

The initial energy distribution of the secondary electrons is assumed to be Gaussian with an rms value of $5 \mathrm{eV}$, and their initial angular distribution corresponds to a $\cos \theta$ distribution in spherical coordinates.

Beam fields are calculated using the standard expression à la Bassetti-Erskine for a Gaussian bunch transverse population [24] or the simpler formula for round beams. An elegant expression for the field at large distances which

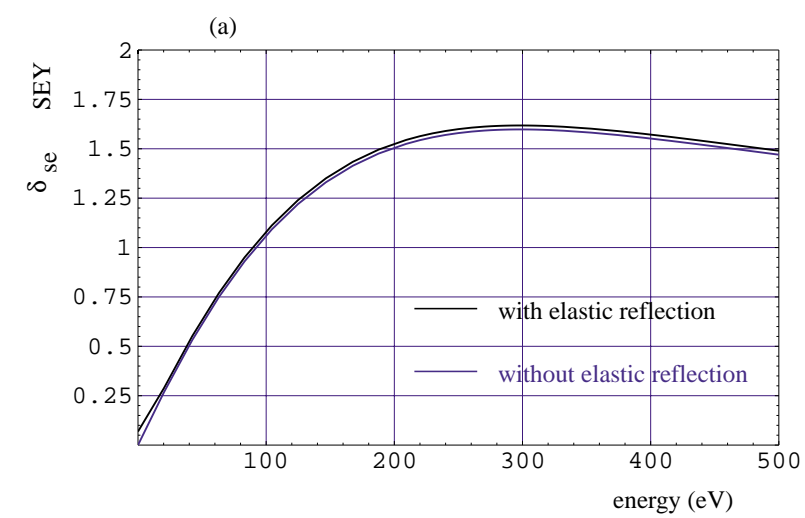

(b)

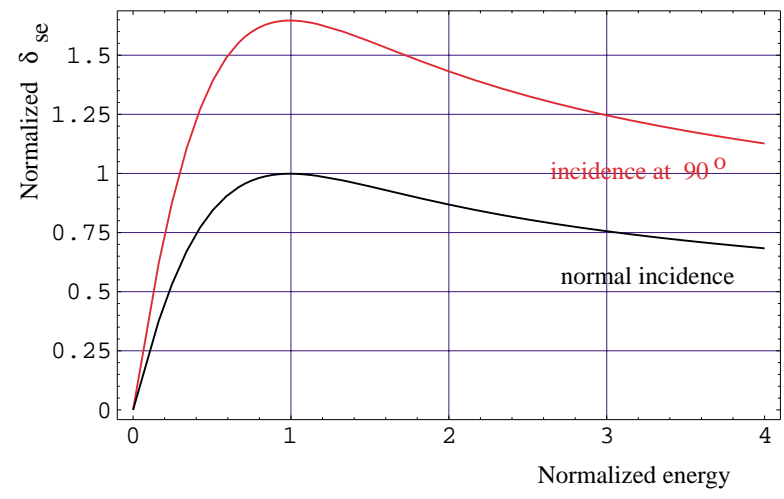

FIG. 7. (Color) (a) Secondary emission yield ( $\delta_{\mathrm{se}}$ and SEY $=$ $\left.\delta_{\mathrm{se}}+\delta_{e}\right)$ vs primary electron energy $E_{p}$, for $\delta_{\max }=1.6$ and $E_{\max }=300 \mathrm{eV}$ with and without elastic scattering. (b) Normalized secondary emission yield $\delta_{\text {se }} / \delta_{\max }$ vs normalized primary electron energy $E_{p} / \epsilon_{\max }$ for two different angles of incidence.

includes the image charges in an elliptical chamber was given by Furman and Lambertson [23], and implemented for LHC electron-cloud simulations by Brüning [11]. Denoting by $\mathcal{E}=\mathcal{E}_{x}+i \mathcal{E}_{y}$ the complex electric field, Furman's expression reads [23]

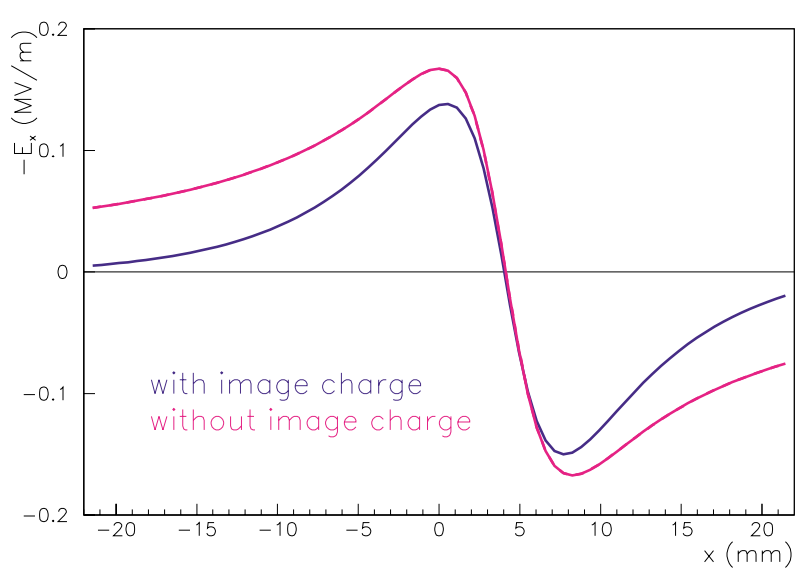

FIG. 8. (Color) Horizontal electric field vs horizontal position at $y=0$ for an elliptical chamber with $22 \times 10 \mathrm{~mm}$ half-apertures and a beam offset of $4.3 \mathrm{~mm}$ in both transverse directions. 


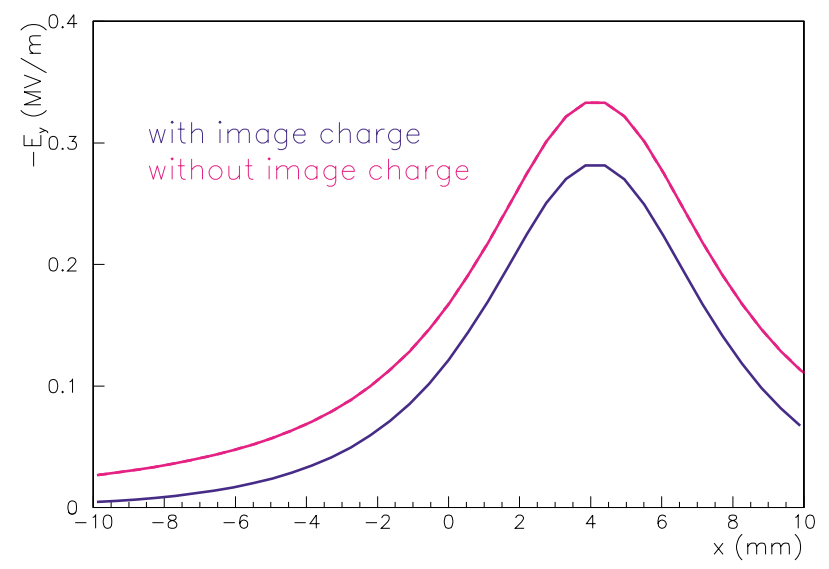

FIG. 9. (Color) Vertical electric field vs horizontal position at $y=0$ for an elliptical chamber with $22 \times 10 \mathrm{~mm}$ half-apertures and a beam offset of $4.3 \mathrm{~mm}$ in both transverse directions.

$$
\begin{aligned}
& \mathcal{E} \approx \frac{2}{\bar{z}-\bar{z}_{0}} \\
&+\frac{4}{g} \sum_{n=1}^{\infty} e^{-n \mu_{c}}\left[\frac{\cosh n \mu_{0} \cos n \phi_{0}}{\cosh n \mu_{c}}\right. \\
&\left.+i \frac{\sinh n \mu_{0} \sin n \phi_{0}}{\sinh n \mu_{c}}\right] \frac{\sinh n \bar{q}}{\sinh \bar{q}}
\end{aligned}
$$

where $z=x+i y=g \cosh q=g \cosh (\mu+i \phi) \quad$ denotes the test position, $z_{0}=x_{0}+i y_{0}=g \cosh q_{0}=$ $g \cosh \left(\mu_{0}+i \phi_{0}\right)$ denotes the position of the source, and both $g=\sqrt{a^{2}-b^{2}}$ and $\mu_{c}=\tanh ^{-1}(b / a)$ characterize the vacuum chamber with semiaxes $a$ and $b$. In the simulation, the infinite sum is truncated at an adjustable order $n$ (for instance, $n=30$ ).

Figures 8 and 9 depict the horizontal and vertical electric fields for an offset beam as a function of horizontal position, again with and without including the field from the

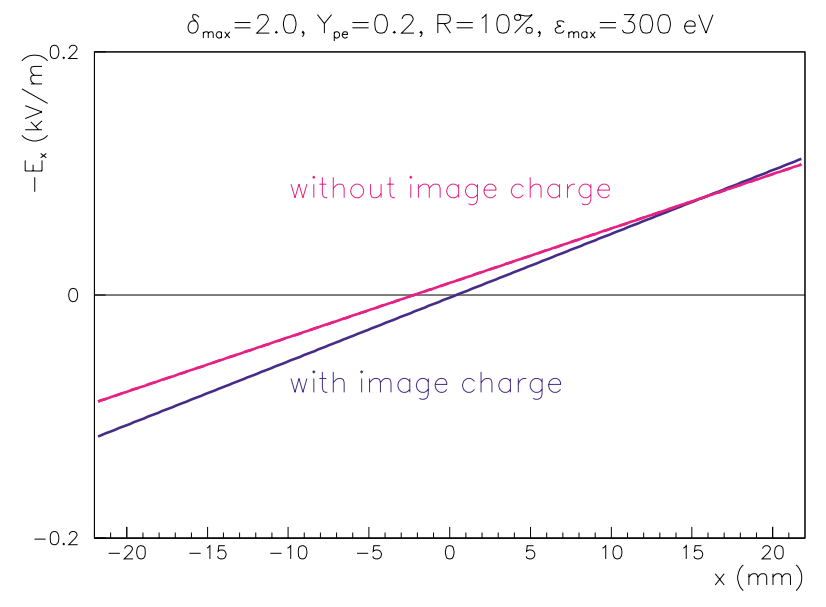

FIG. 10. (Color) Horizontal electric space-charge field of electron cloud vs horizontal position after the passage of eight bunches in the LHC. Parameters: $\delta_{\max }=2.0, Y_{p e}=0.2, R=$ 0.1 , and $\epsilon_{\max }=300 \mathrm{eV}$. image charges. Both demonstrate that the image charges can significantly alter the electron motion. Image charges of the electron cloud are also taken into account. The electron charges are assigned to points on a grid, typically consisting of $25 \times 25$ points, and the image forces are evaluated for each of the grid points. An example of the electron-cloud self-field with and without image charges is shown in Fig. 10.

\section{A. Heat load on the LHC beam screen}

As the first example of application, we are going to evaluate the heat load on the beam screen of LHC (see Table I for an overview on important beam and machine parameters). First analytical estimates are actually based on the following parameters and assumptions [9]. Referring to Eq. (1), one can easily evaluate that for the LHC at $7 \mathrm{TeV}$ the linear photon flux is approximately $10^{17}$ photons per second and meter; neglecting secondary emission and photon reflection, the linear heat load is given by

$$
\langle P\rangle=\left\langle E_{p e}\right\rangle Y 10^{17} \frac{\mathrm{eV}}{\mathrm{sm}},
$$

where $\left\langle E_{p e}\right\rangle$ is the average electron energy. Thus, with $Y=0.02$ one gets approximately $2 \times 10^{15}$ photoelectrons per second and per meter. Assuming furthermore a uniform electron cloud in the vacuum chamber, the average energy gain after a bunch passage per electron is approximately $700 \mathrm{eV}$ [12]. Plugging these numbers into Eq. (8), we get a linear heat flow of

$$
\langle P\rangle \approx 0.2 \mathrm{~W} / \mathrm{m} \text {. }
$$

However, it is clear that the assumption of a uniform electron distribution is not justified, and therefore more precise estimations need to be made by means of a dedicated simulation study also including secondary emission. Figure 11 shows that for the LHC dipole chamber the so-called critical value of the secondary emission yield, above which an exponential growth of the number of electrons occurs [11], lies certainly between 1.1 and 1.5 . In fact, one can check that for $\delta_{\max }=1.3$ already an unabated growth of the electron cloud is observed when space charge from the electrons is artificially switched off. For $\delta_{\max }>1.3$, the

TABLE I. Overview of LHC parameters essential for the simulation.

\begin{tabular}{lc}
\hline \hline Circumference & $26658.883 \mathrm{~m}$ \\
Relativistic $\gamma$ & 7461 \\
Bunch population & $1.05 \times 10^{11} e^{+}$ \\
Physical emittances $\left(\epsilon_{x, y}\right)$ & $7.82,0.503 \mathrm{~nm} \mathrm{rad}$ \\
Tunes $\left(Q_{x, y, s}\right)$ & $63.28,63.31,0.00212$ \\
rms beam sizes $\left(\sigma_{x, y, z}\right)$ & $0.303,0.303,77 \mathrm{~mm}$ \\
Spacing between bunches & $7.48 \mathrm{~m}$ \\
rms energy spread & 0.111 \\
Mom. compaction factor & $\alpha=3.47 \times 10^{-4}$ \\
Horizontal and vertical half-apertures & $2.2,1.8 \mathrm{~mm}$ \\
\hline \hline
\end{tabular}




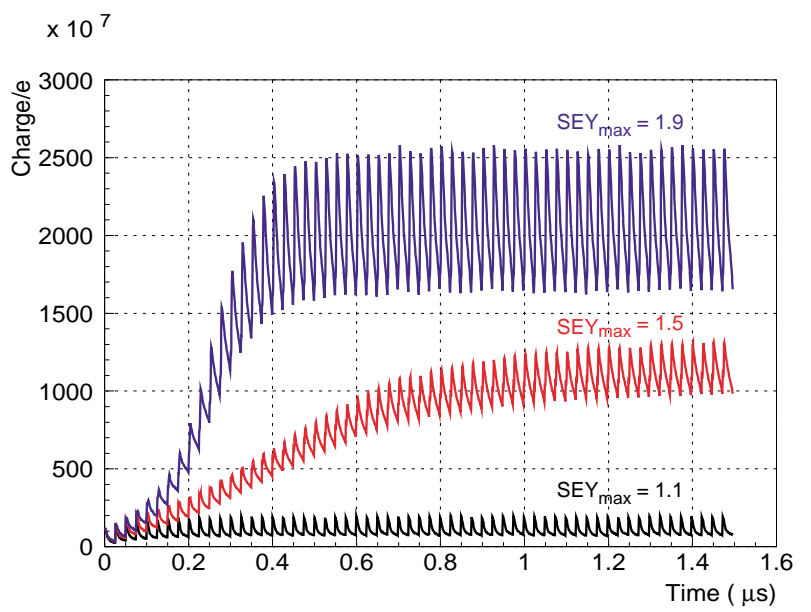

FIG. 11. (Color) Electron-cloud build up in the bending section of the LHC for three different values of the maximum secondary emission yield $\left(N_{b}=1.05 \times 10^{11}\right.$ protons/bunch and dipole length $14.2 \mathrm{~m})$.

electron-cloud build up does not continue forever, but it is ultimately stopped by the repelling self-field of the electron cloud. Figure 11 clearly shows the saturation of the build up, which takes longer for $\delta_{\max }=1.5$ but occurs after only a few bunches when $\delta_{\max }=1.9$, at a line density of a few $10^{9}$ per meter.

The electron cloud is neither concentrated around the beam nor spread out uniformly inside the chamber. In the dipole magnets, and especially if the secondary emission yield is larger than the critical value, two vertical stripes with high electron density surround the beam, as illustrated in Fig. 12 for the LHC bending magnet. The two stripes represent regions at which the average energy gain from the beam is about equal to the energy $\epsilon_{\max }$ of maximum secondary emission.

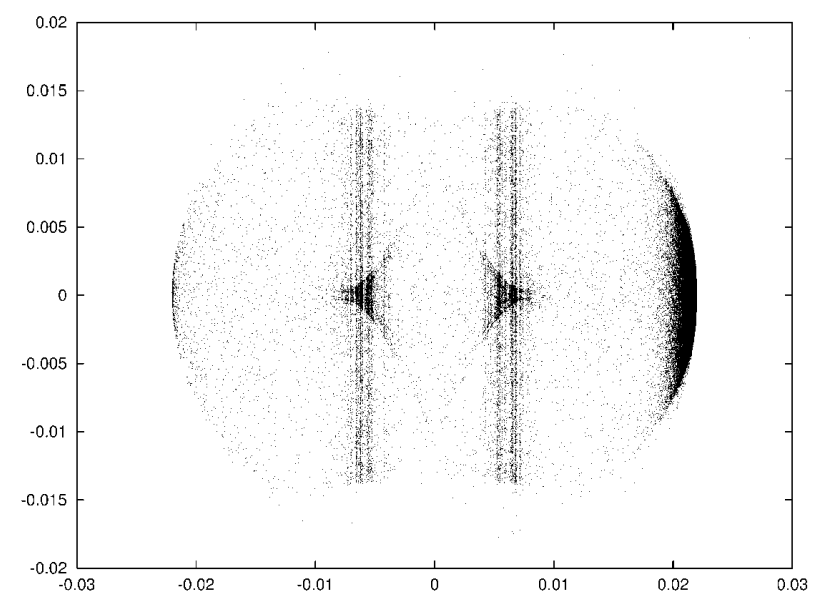

FIG. 12. Snapshot of transverse electron-cloud distribution in an LHC dipole chamber after 60 bunches with the design current. Vertical stripes indicate regions with large secondary emission. Parameters: $\mathrm{SEY}_{\max }=1.3, \epsilon_{\max }=450 \mathrm{eV}, R=0.1$, and $Y^{*}=0.025$.
For the LHC the heat load from the incident electrons is a concern. The LHC cryogenic system is designed for a maximum beam screen heat load of about $1 \mathrm{~W} / \mathrm{m}$. Since the resistive heating by the beam and synchrotron radiation both amount to about $0.2 \mathrm{~W} / \mathrm{m}$, the average heat load due to the electron cloud must be smaller than $0.6 \mathrm{~W} / \mathrm{m}$.

In Fig. 13 the average heat load over a train of 60 bunches is plotted as a function of the maximum secondary emission yield. In both diagrams, one can observe that the slope of the curve increases in the neighborhood of the critical yield, between 1.2 and 1.3. It is clear from the upper diagram of Fig. 13, and from comparison with previous calculations made with a uniform distribution of $10 \%$ reflected light, that the heat load on the beam screen is not highly affected by the exact shape of the distribution of the reflected photons. If we account for the contribution of elastic scattering when the electrons hit the wall [according to Eq. (6)], we can see from the lower diagram in Fig. 13 that the average heat load increases by only about $10 \%$ for the highest SEYs. Evaluations of the heat load for different values of the parameters used in Eq. (6) should be made in
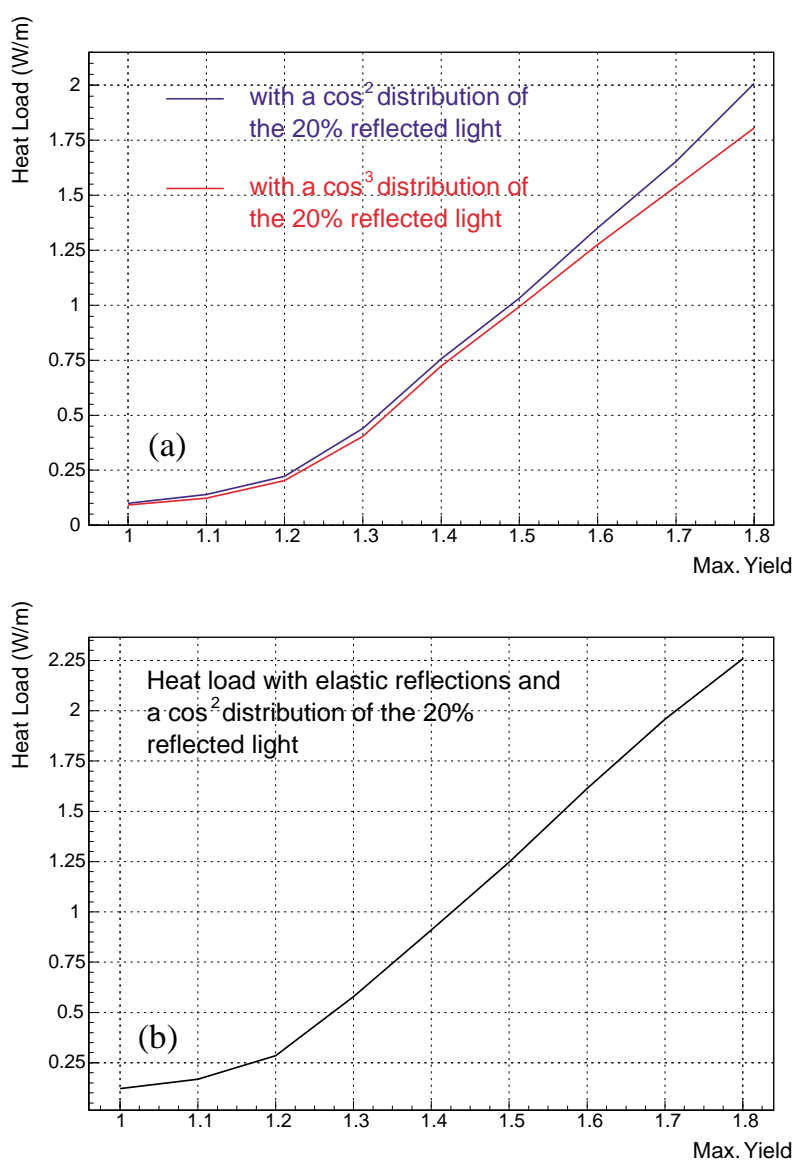

FIG. 13. (Color) Heat load in the LHC dipole chamber vs maximum secondary emission yield. Diagram (a) shows the heat load evaluation for two different distributions of the reflected light but without accounting for the elastic scattering of the electrons against the beam pipe; in (b) the contribution of elastic scattering is included according to the modeling in Eq. (6). 
order to ensure that the effect of elastic reflections can be really considered small in all cases. Measurements carried out this year seem to suggest that better values to be used for LHC would be $\hat{\delta}_{e}=0.56, \delta_{e, \infty}=0, \Delta=52 \mathrm{eV}$, and $E_{e}=0$ [25]. Simulations with the new parameters are presently being performed, and preliminary results suggest that the contribution of elastic scattering might significantly increase the heat load on the beam screen.

\section{B. Electron-cloud effects on the LHC beam position monitors}

One of the concerns related to the presence of an electron cloud in the beam pipe is the way the electrons can affect the correct functioning of beam position monitors placed along the beam orbit. The electrons hitting the plates of the monitor cause a net flow of charge between the electrodes, and consequently a nonzero current signal flowing through the plates. An example of the transverse section of a beam position monitor is sketched in Fig. 14: the beam pipe is $48 \mathrm{~mm}$ wide and the whole device stretches over a length of $24 \mathrm{~mm}$. Multipacting between the pickup electrodes is indeed possible, and it actually occurs even for very low values of the secondary emission yield (a radius of about $3 \mathrm{~cm}$ is in fact "the worst case," as shown in simulations [26] or using a semianalytical formula [27]).

In order to evaluate the effects of the electron cloud on the signal detected by the monitor, we have subdivided the pipe section into four parts corresponding to the four electrodes, as illustrated in Fig. 14, and we have let the simulation update at each time step the net amount of charge and electric current going in or out of the electrode associated to each of them. In Fig. 15, we can clearly see that the electrons are mostly emitted at the first electrode, consistently with the fact that in the simulation we have

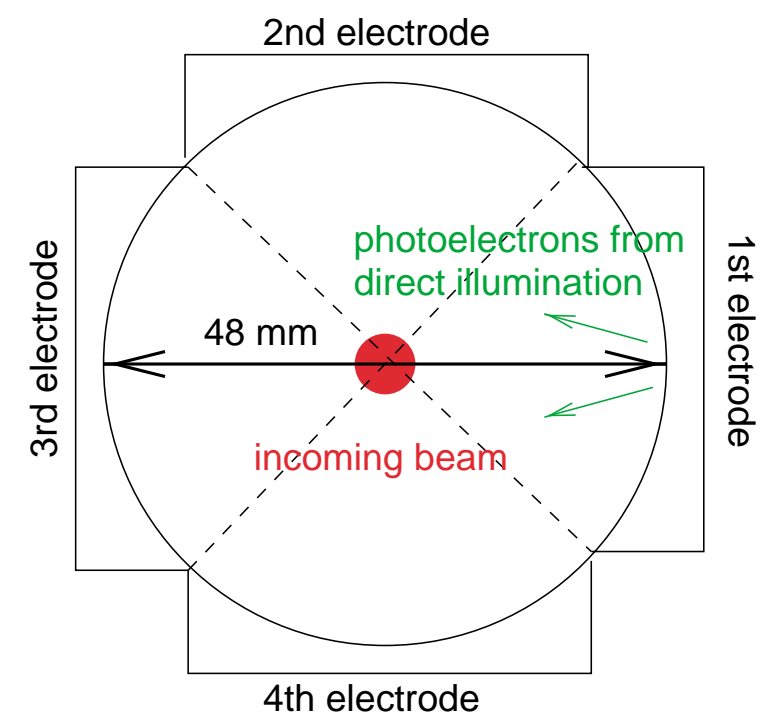

FIG. 14. (Color) Transverse section of an LHC beam position monitor. The incoming beam directly illuminates the first electrode. The device has a longitudinal extension of $24 \mathrm{~mm}$. assumed only $10 \%$ of reflected photons, and that therefore $90 \%$ of the photons cause primary emission within a small area belonging to this first electrode. The electrons subsequently hit the wall again quite uniformly in the transverse azimuthal coordinate. When the maximum secondary emission yield is low, the first electrode alone emits electrons regularly, whereas all the electrodes receive them
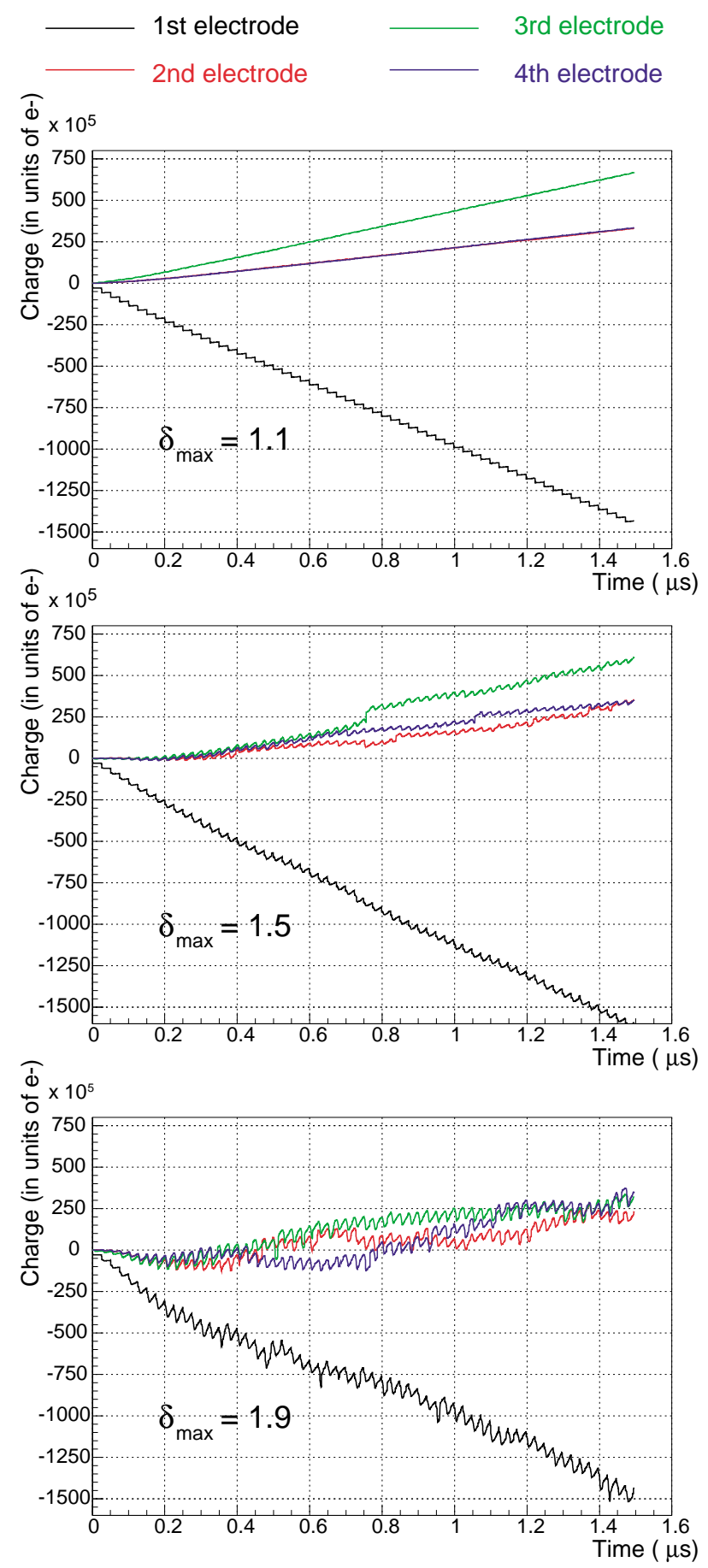

FIG. 15. (Color) Net charge deposited or emitted at each BPM electrode. Here we used the convention that negative values mean a prevalence of outgoing electrons at that plate (net current into the plate). 
as regularly. Therefore, the number of electrons going out of the first electrode grows linearly, and, correspondingly, the amount of charge deposited on the other electrodes also increases linearly (see the first of Figs. 15). When higher values of SEY are considered, it remains basically unchanged that from the first electrode there is a fairly regular emission of electrons all through the 60 bunch passages, but the situation at the other plates becomes progressively more chaotic, because secondary emission with possibly high yield values gives rise to net fluxes that can be in whichever direction, depending upon the properties of the hitting electrons (see second and third diagrams in Fig. 15). The total net amount of electrons flowing through one plate per bunch passage (about $10^{6}$ in the worst case, as one can easily deduce from the diagrams in Fig. 15, taking into account that the total simulation time corresponds to 60 bunch passages) should not seriously affect the resolution of the beam position monitor [28].

In Fig. 16, as an example, the time profile of the current signal due to electrons on the first electrode is plotted in the upper diagram, and its Fourier spectrum in the lower one. The set of unevenly sampled data provided by the simulation (which, for computational needs, makes use of

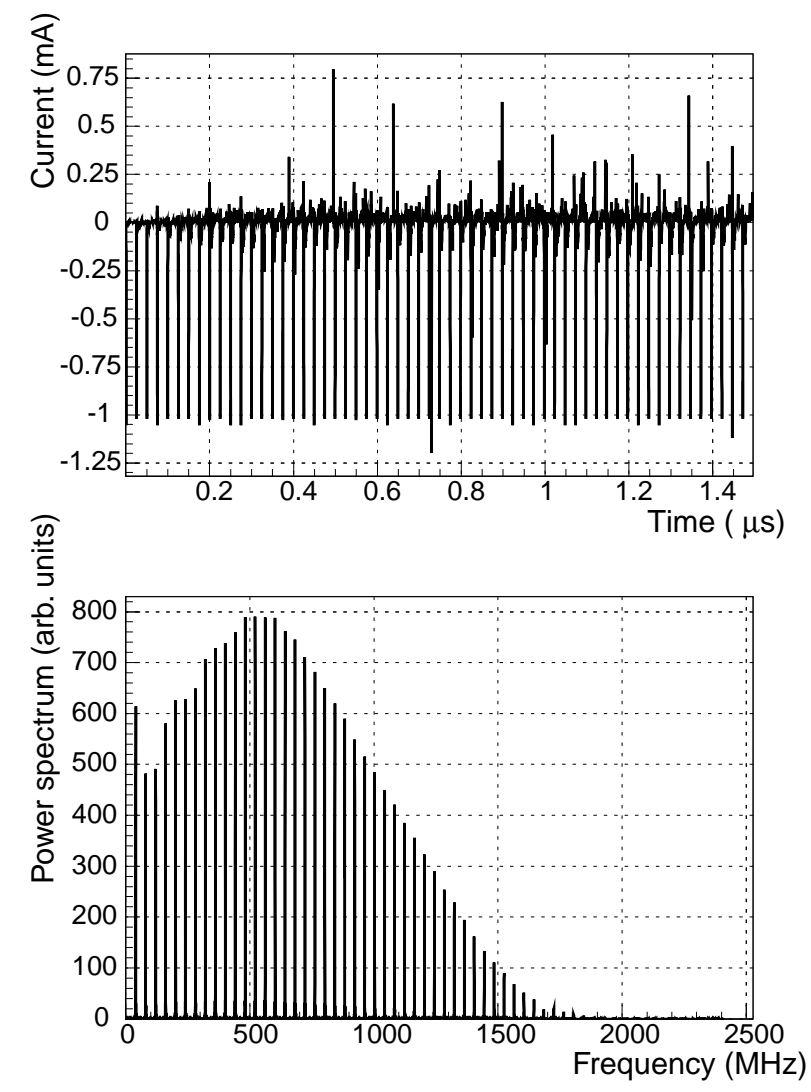

FIG. 16. Current flowing to the first electrode of the BPMs when the maximum secondary emission yield is assumed to be 1.5. In the upper picture one can see the time profile of the current signal (mainly dominated by negative pulses at the bunch frequency, due to photoemission), whereas in the lower picture its power density spectrum is plotted. two different time steps according to whether the bunch is passing and generating new photoelectrons or the electrons are simply evolving in the gap between two subsequent bunches) has been Fourier analyzed with the Lomb algorithm for sequences of unequally spaced samples [29]. If there were only emitted electrons due to the illuminating passing bunch, we would expect the spectrum to be a series of peaks at multiples of $40 \mathrm{MHz}$, enveloped by a Gaussian curve representing the spectrum of the single time pulse (having the bunch shape); it is likely that the flux of electrons which steadily deposit on this electrode can create a distortion from this ideal shape at the low frequencies.

However, the spectra of the current signals show in general that there are coherent currents at the BPM plates, which extend in frequency up to $2 \mathrm{GHz}$ and have strong components centered at multiples of the bunch frequency $40 \mathrm{MHz}$.

\section{ELECTRON-CLOUD INDUCED SINGLE BUNCH INSTABILITIES}

A uniformly distributed electron cloud all inside the beam pipe can be responsible for bunch instabilities. Multibunch instabilities were first observed at the KEK Photon Factory [3] and are already widely investigated by means of computer simulation: if a bunch is sent through offset with respect to the other bunches, it will perturb the electron-cloud distribution and the next bunch will receive an additional deflection caused by this perturbation. Growth times of this type of instability have been estimated using an analytical formula [30] with the bunch-to-bunch wakefield obtained from the electroncloud simulation $[4,6,8,26]$. As the instability is very slow for the present LHC parameters (rise times longer than $1 \mathrm{~s}$ ), it is expected that Landau damping by the natural intrabunch tune spread can counterbalance it before it damages the bunch structure and leads to beam loss.

The electron cloud can also act as a short-range wakefield and drive a single bunch instability [31,32]. Such kinds of instability could be responsible for the vertical emittance blow up that is observed at the KEK B Factory [33], and possibly also for that in SPS. In the following, we present the model that we have employed to simulate single bunch effects due to the electron cloud and some results of its application to the low energy ring of the KEK B Factory.

\section{A. Model}

A proton or positron bunch interacts with the electron cloud during its passage. When we study the single-bunch effects of the electron cloud, only perturbations of the cloud induced by the passing bunch are considered. All the relevant bunch and lattice parameters, as well as the average equilibrium density of the electron cloud along the ring, are basic input parameters for the simulation of the coupled motion between bunch and cloud electrons. 
For simplicity, the kick approximation is used for the action of the electron cloud on the bunch: the cloud is assumed to be localized at one or more definite positions along the ring, $s=n s_{\text {el }}$ with $n=0,1, \ldots,\left(N_{\text {int }}-1\right)$. Both the cloud and the bunch are modeled as ensembles of macroparticles (with $N_{p}$ bunch macroparticles and $N_{e}$ macroelectrons in the cloud). The bunch is also divided into $N_{\mathrm{sl}}$ slices, which interact with the electron cloud after one another and cause the distortion of the initially uniform cloud distribution that can significantly affect the part of the bunch coming later. The principle of the simulation is synthetically illustrated in Fig. 17. The interaction between bunch particles and cloud electrons is expressed by the equations of motion

$$
\frac{d^{2} \underline{x}_{p, i}(s)}{d s^{2}}+\underline{\underline{K}}(s) \underline{x}_{p, i}(s)=\frac{2 r_{e} N_{\mathrm{eme}}}{\gamma} \sum_{j=1}^{N_{\mathrm{el}}} \sum_{n=0}^{N_{\mathrm{int}}-1} \underline{F}\left[\underline{x}_{p, i}(s)-\underline{x}_{e, j} ; \sigma_{\mathrm{me}}\right] \delta\left(s-n s_{\mathrm{el}}\right),
$$

$$
\frac{d^{2} \underline{x}_{e, j}}{d t^{2}}=-\left(2 r_{e} c^{2}\right) \sum_{k=1}^{N_{\mathrm{sl}}} N_{\mathrm{p} k} \underline{F}\left(\underline{x}_{e, j}-\underline{x}_{\mathrm{off} k} ; \sigma_{k}\right),
$$

where the positions of electrons and bunch particles are represented by the vectors $\underline{x}_{e} \equiv\left(x_{e}, y_{e}\right)$ and $\underline{x}_{p}(s) \equiv$ $\left(x_{p}, y_{p}, z_{p}\right), z=s-c t$ being a comoving longitudinal coordinate; $\underline{\underline{K}}(s)$ is the transfer matrix with the focusing strengths between two interaction points; $N_{\mathrm{eme}}$ and $N_{\mathrm{p} k}$ represent the number of electrons in one macroelectron and the number of particles in the $k$ th bunch slice, respectively; $\underline{x}_{\text {off } k}$ and $\sigma_{k}$ are the transverse offset and the rms size of the $k$ th slice; $\sigma_{\mathrm{me}}$ is the rms size of a macroelectron, which is assumed to have finite size (typically a tenth of the beam size); $\underline{F}$ is expressed by the Bassetti-Erskine formula [24]. The technique of using macroparticles with finite size is not new in the framework of plasma physics

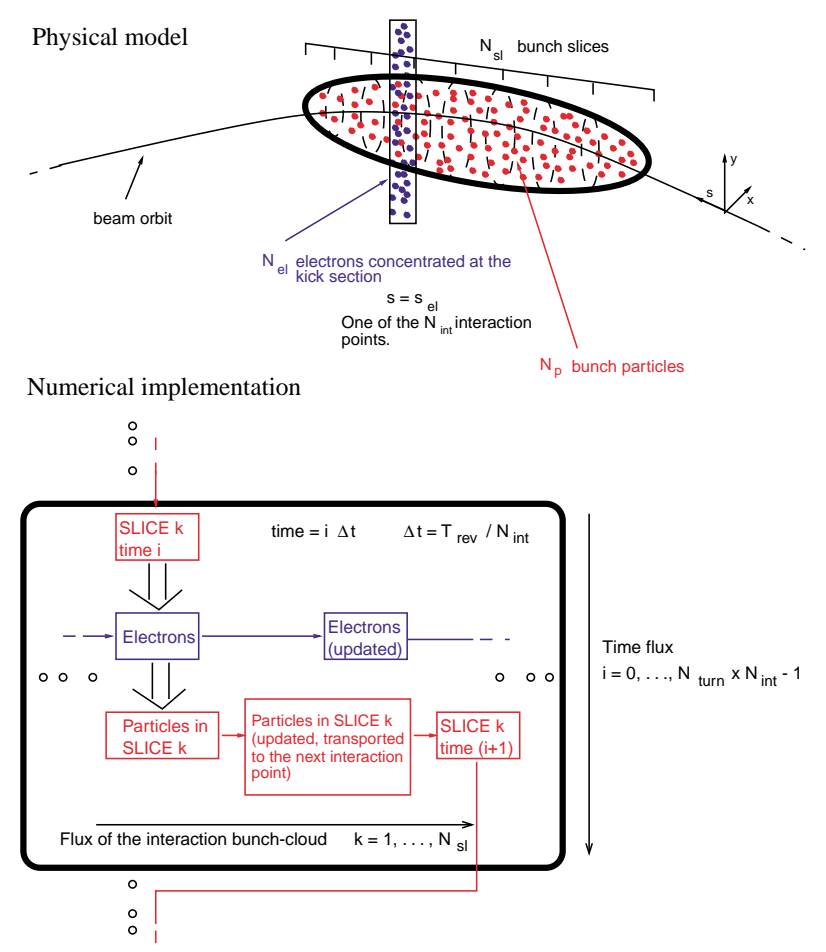

FIG. 17. (Color) Schematic of the simulation program for single-bunch instability.
[34,35] and beam-beam simulations for linear collider, where its purpose is to avoid singularities which may invalidate the simulations while retaining the long-range behavior of the fields that are responsible for collective effects. We have chosen to use elliptical macroelectrons, and the rms sizes of each have been calculated from its "natural" transverse area (namely, the area transversely occupied by the total ensemble divided by the overall number of macroelectrons) with the assumption that their ratio equals the ratio between bunch transverse rms sizes. These numbers have been further scaled down by an appropriate factor in order to account for Gaussian distributions. Adjusting this factor we ran the code for different macroelectron sizes, and verified that the influence of this parameter on the results of the simulations is in fact negligible, as long as the electrons stay much smaller than the beam transverse size but big enough that the probability of large kicks remains also low.

The interaction between bunch and electron cloud is simulated following the steps that are shown in Fig. 17. The macroelectrons have initially a uniform distribution which extends transversely over a region 10-20 times larger than the bunch rms sizes. The initial velocities of the macroelectrons are set to 0 . The particles in the bunch are set to have initial Gaussian distributions in each coordinate of their six-dimensional phase space, and the bunch is subsequently sliced. The bunch slicing is actually repeated each time the interaction starts, because when synchrotron motion is taken into account, particles mix longitudinally. As the bunch slice $k$ interacts with the electron cloud concentrated at the kick point, the particles therein contained and the electrons receive a mutual kick; the perturbed electron cloud acts then on the particles in the next bunch slice $k+1$, whereas the slice $k$ will be newly kicked by the electron cloud at the next interaction point (after all the bunch particles have been propagated using a linear transport matrix, which can optionally account for the synchrotron motion, and have received a chromaticity kick). Without synchrotron motion, the mechanism does not affect the head of the bunch, which always feels a zero total force from the electrons, but it can drive its tail unstable. The electron-cloud configuration is uniformly regenerated at the beginning of each interaction. 
In our simulations, we have used $10^{4}$ macroelectrons, and the same number of macroparticles for the bunch. The bunch has been divided into 20-30 slices. The interaction starts from the slice containing particles with the largest positive values of $z$. The model that we have used allows us to resolve the head-tail motion of the bunch, as well as the horizontal and vertical emittance and rms size evolutions, both locally and averaged over the full bunch. Similar simulations were reported in [32], where the beam was represented by an ensemble of microbunches with constant transverse size.

\section{B. Results}

Using the model previously introduced, hereafter we present computer simulations of beam instability for the low energy ring of the KEK B Factory. Typical parameters that have been used for this study are summarized in Table II. Different values for the average electron cloud density along the ring $\left(n_{\mathrm{el}}\right)$ have been used, ranging from $2 \times 10^{11} \mathrm{~m}^{-3}$ to $10^{12} \mathrm{~m}^{-3}$ (as results from the simulations of electron-cloud build up [32]).

Figure 18 shows the time evolution of horizontal and vertical positions of the beam centroid over 100 turns for a set of three different cases, and an electron cloud density $n_{\mathrm{el}}=10^{12} \mathrm{~m}^{-3}$. One can see that, while in the horizontal direction no major differences occur and the oscillation of the centroid always stays at a level of about $2 \%$ of the original bunch rms size, in the vertical direction a growing amplitude of the oscillation is clearly visible for the case where synchrotron motion has been artificially switched off. Synchrotron motion always reduces the amplitude of the oscillations due to particles mixing in the longitudinal direction.

Taking a look at the detailed pictures showing how the bunch vertical displacement is actually distributed along the bunch at subsequent times, one can immediately recognize a vertical head-tail motion that goes unstable (Fig. 19). A longer overview on the time evolution of the vertical beam offset (over 600 turns in Fig. 20) shows that the unstable motion saturates after 100-200 turns, and

TABLE II. Overview of KEK B LER parameters essential for the simulation.

\begin{tabular}{lc}
\hline \hline Circumference & $3016 \mathrm{~m}$ \\
Relativistic $\gamma$ & 7000 \\
Bunch population & $3.3 \times 10^{10} e^{+}$ \\
Emittances $\left(\epsilon_{x, y}\right)$ & $1.8 \times 10^{-8}, 3.6 \times 10^{-10} \mathrm{~m}$ \\
Tunes $\left(Q_{x, y, s}\right)$ & $45.53,44.11,0.015$ \\
rms beam sizes $\left(\sigma_{x, y, z}\right)$ & $0.42,0.06,4 \mathrm{~mm}$ \\
Average beta functions & $\beta_{x, y}=10 \mathrm{~m}$ \\
rms energy spread & 0.0007 \\
Mom. compaction factor & $\alpha=1.8 \times 10^{-4}$ \\
Chromaticities $\left(Q_{x, y}^{\prime}\right)$ & 4,8 \\
\hline \hline
\end{tabular}
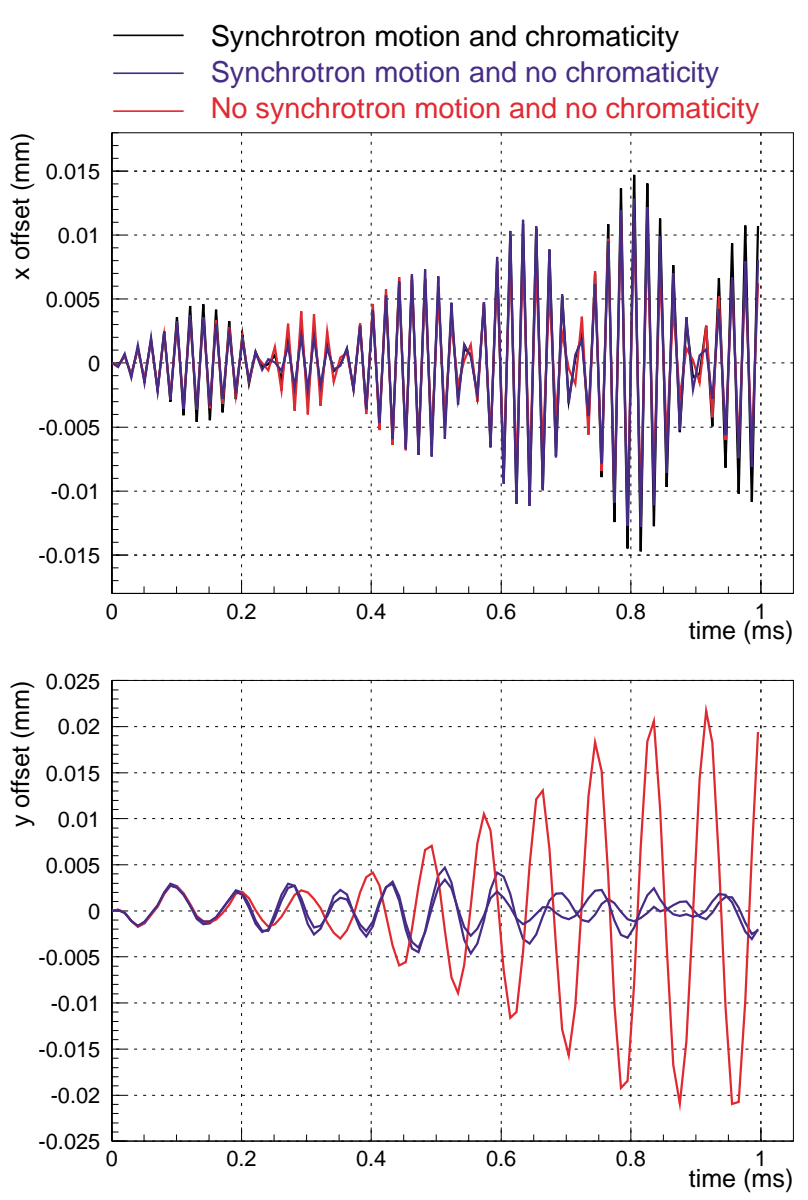

FIG. 18. (Color) Time profiles of the horizontal and vertical average position of the bunch centroids for the first 100 turns.

then enters a nonlinear phase in which a coherent oscillation at quite large amplitudes appears to be surviving but no further growth occurs. Figure 20(b), which refers to a lower electron cloud density, shows even more clearly the exponential character of the initial growth; on the other hand, here the rise time is larger, and the peak value eventually reached by the oscillation smaller, because of the weaker field felt by the bunch particles.

In Fig. 21, the time evolutions of horizontal and vertical bunch rms sizes and emittances for the same cases as in Fig. 18 are plotted. Again, while just small amplitude oscillations are to be observed in the horizontal direction, a constant increase is present in the vertical direction, which is smaller when the synchrotron motion of the positrons is accounted for. In the long run, we can see that the vertical emittance even doubles its initial value after about 550 turns for the case with zero chromaticities and without synchrotron motion, and that it increases linearly, getting $10 \%$ of its initial value every 500 turns roughly, when synchrotron motion is switched on; the horizontal emittance is subject to minor changes, and grows in fact no higher than its nominal value $1.8 \times 10^{-7} \mathrm{~m}$ (Fig. 22). 

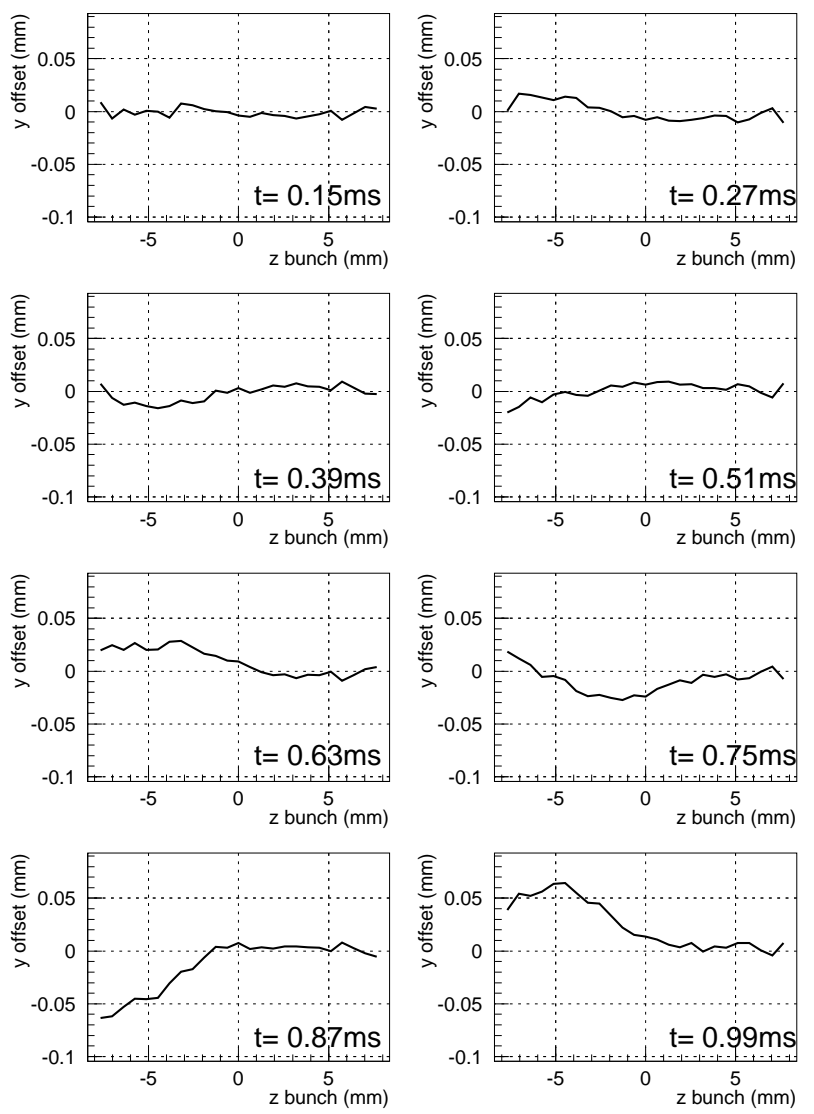

FIG. 19. Snapshots of the vertical centroid position along the bunch, when synchrotron motion is not taken into account and both chromaticities are set to 0 .

\section{CONCLUSIONS}

In this paper we have reported about the model for electron-cloud simulations and we have shown some of its more recent applications to LHC, such as the heat load estimation taking into account elastic reflection of the electrons at the beam pipe wall and the possible influence of the electrons on the instrumentation for diagnostics. New estimations of the heat load with elastic scattering have to be carried out, using a revised set of parameters resulting from the most recent measurements on prototype LHC vacuum chambers.

In the second part of the paper, we discussed a second macroparticle model for the description of the electroncloud single-bunch interaction, in order to study numerically phenomena such as single-bunch instability driven by the electron cloud. First results of this type of simulation, applied to the KEK B LER, were presented in the last section, where we described the unstable evolution of the bunch centroid in the vertical direction (the horizontal one does not seem to give rise to any serious unstable motion, at least on the time scale covered by our simulations), and estimated the expected emittance increase. A more detailed analysis of the data resulting from these simulations is foreseen now in order to determine characteristic
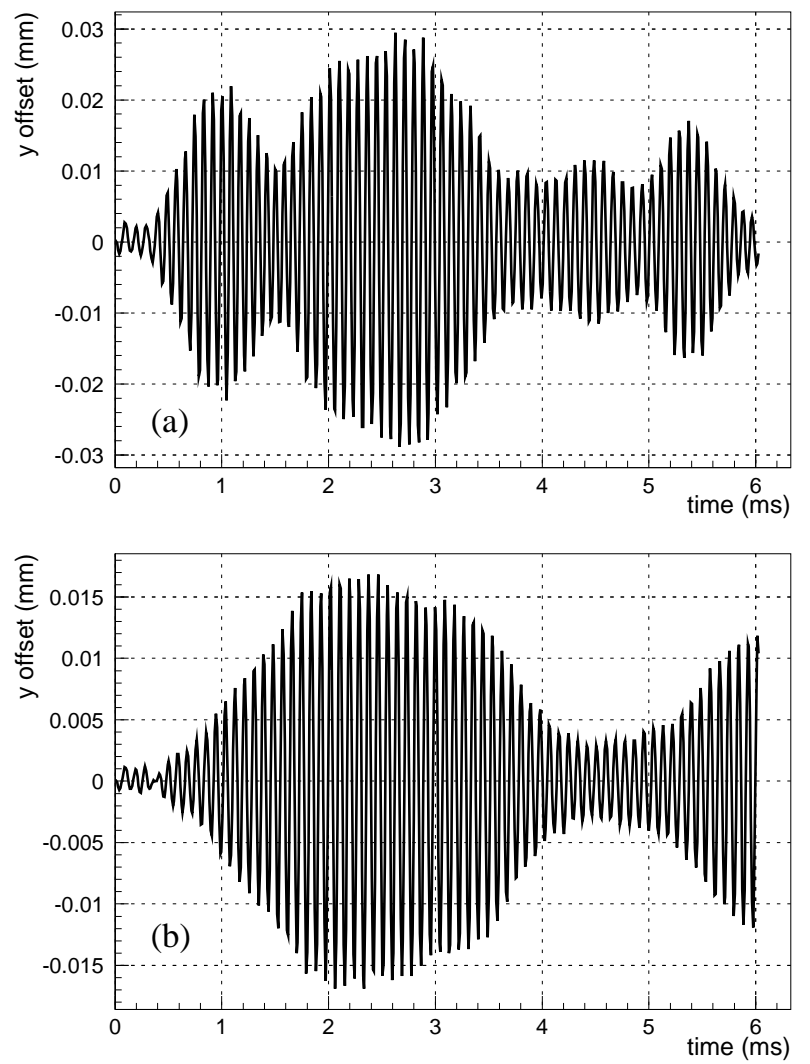

FIG. 20. Time profiles of the vertical average position of the bunch centroid for two different values of the electron-cloud density $\left(10^{12} \mathrm{~m}^{-3}\right.$ for the upper picture and $4 \times 10^{11} \mathrm{~m}^{-3}$ for the lower one).

frequencies and rise times of the instability and to relate them with all the beam and cloud parameters. Analytical work for a complete description of the phenomenon, and application to the SPS and comparison with experimental data of single bunch fast instabilities, will also be carried out in future studies.
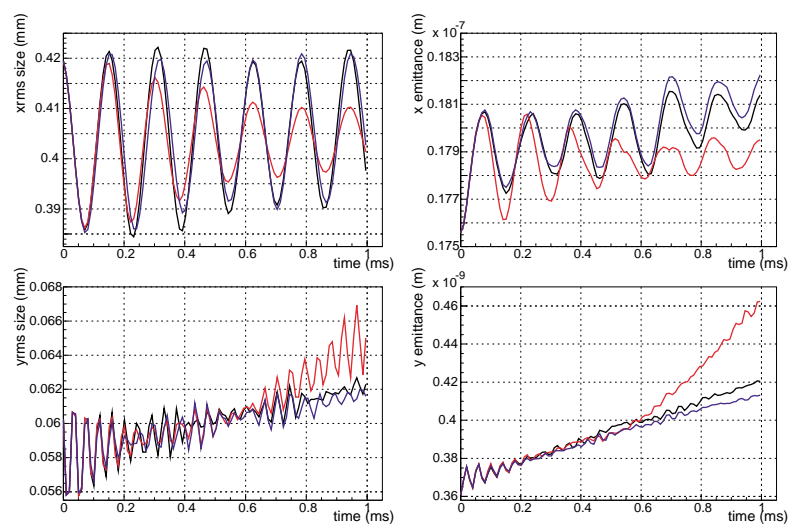

$\widehat{\underline{E}} \times 10^{-9}$

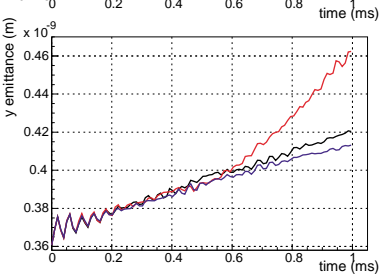

Synchrotron motion and chromaticity

Synchrotron motion and no chromaticity

- No synchrotron motion and no chromaticity

FIG. 21. (Color) Bunch rms sizes and emittances for the same cases as in Fig. 18. 


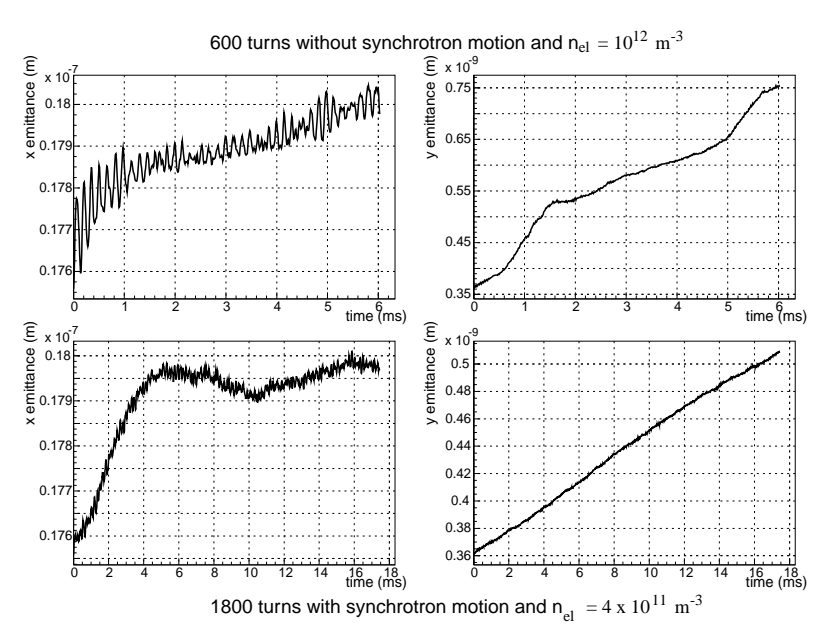

FIG. 22. Time evolutions of horizontal and vertical emittances without synchrotron motion (upper pictures) and with synchrotron motion included (lower pictures).

\section{ACKNOWLEDGMENTS}

The authors would like to thank A. Arauzo Garcia, O. Bruening, F. Caspers, I. Collins, G. Forconi, M. Furman, O. Groebner, R. Jones, K. Ohmi, and A. Rossi for helpful discussion and information.

[1] O. Gröbner, in Proceedings of the 10th International Conference on High Energy Accelerators, Protvino, Russia, 1977 (Institute of High Energy Physics, Protvino, 1977).

[2] O. Gröbner, Vacuum, 47, 591 (1996).

[3] M. Izawa, Y. Sato, and T. Toyomasu, Phys. Rev. Lett. 74, 5044 (1995).

[4] K. Ohmi, Phys. Rev. Lett. 75, 1526 (1995).

[5] Z. Y. Guo et al., in Proceedings of the 1st Asian Particle Accelerator Conference (APAC 98), Tsukuba, Japan, 1998 (KEK Report No. 98-23, 1998).

[6] M. A. Furman and G. R. Lambertson, in Proceedings of the 17th IEEE Particle Accelerator Conference (PAC 97), Vancouver, Canada (IEEE, Piscataway, NJ, 1998).

[7] S. Heifets, SLAC Report No. SLAC-PUB-6956, 1995.

[8] F. Zimmermann, CERN Report No. CERN-LHCPROJECT-REPORT-95, SLAC Report No. SLAC-PUB7425, 1997.

[9] O. Gröbner, Report No. CERN-LHC-PROJECT-REPORT127, 1997.

[10] J. B. Jeanneret, CERN Report No. CERN SL/NOTE 97-48 (AP), 1997.

[11] O. Brüning, CERN Report No. CERN-LHC-PROJECTREPORT-158, 1997.
[12] J.S. Berg, CERN Report No. CERN-LHC-PROJECTNOTE-97, 1997.

[13] O. Brüning, in Proceedings of the 1998 European Particle Accelerator Conference (EPAC98), Stockholm (Institute of Physics, Bristol, UK, 1998).

[14] M. Furman, CERN Report No. CERN-LHC-PROJECTREPORT-180, 1998.

[15] L. Vos, CERN Report No. CERN-LHC-PROJECT-NOTE150, 1998.

[16] Proceedings of the Workshop on LEP-SPS PerformanceChamonix X, Chamonix, 2000, edited by P. Le Roux, J. Poole, and M. Truchet (CERN Report No. CERN-SL2000-007 DI, 2000), pp. 110-157.

[17] M. Sands, SLAC Report No. SLAC-0121, 1970.

[18] J. Kouptsidis and G. A. Mathewson, DESY Internal Report No. DESY 76/49, 1976 (unpublished).

[19] F. Zimmermann, Nucl. Instrum. Methods Phys. Res., Sect. A 398, 131 (1997).

[20] I. Collins (private communication).

[21] O. Brüning et al., in Proceedings of the 1999 IEEE Particle Accelerator Conference (PAC99), New York (IEEE, Piscataway, NJ, 1999).

[22] H. Seiler, J. Appl. Phys. 54, 11 (1983).

[23] M. A. Furman and G. R. Lambertson, in Proceedings of the International Workshop on Multibunch Instabilities in Future Electron and Positron Accelerators, Tsukuba, 1997, edited by Y.H. Chin (KEK Report No. 97-17, 1997), p. 170.

[24] M. Bassetti and G. A. Erskine, CERN Report No. CERNISR-TH/80-06, 1980.

[25] I. Collins (private communication).

[26] F. Zimmermann, in Proceedings of the Workshop on LEP-SPS Performance-Chamonix X, Chamonix, 2000 (Ref. [16]), p. 136.

[27] G. Stupakov, CERN Report No. CERN-LHC-PROJECTNOTE-141, 1997.

[28] R. Jones (private communication).

[29] W. H. Press, S. A. Teukolsky, W. T. Vetterling, and B. P. Flannery, Numerical Recipes in $C$ (Cambridge University, Cambridge, England, 1988).

[30] A. W. Chao, Physics of Collective Beam Instabilities in High Energy Accelerators (Wiley, New York, 1993).

[31] F. Zimmermann, CERN Report No. CERN SL-Note2000-004 AP, 2000.

[32] K. Ohmi and F. Zimmermann, CERN Report No. CERN-SL-Note-2000-015 AP, 2000.

[33] H. Fukuma et al., in Proceedings of the European Particle Accelerator Conference, Vienna, 2000, http://accelconf. web.cern.ch/AccelConf/e00/index.html

[34] J. M. Dawson, Rev. Mod. Phys. 55, 403 (1983).

[35] R. W. Hockney and S. W. Eastwood, Computer Simulation Using Particle (Hilger, Bristol, UK, 1988). 